\title{
NUCLEARITY IN AXIOMATIC POTENTIAL THEORY ${ }^{1}$
}

\author{
BY BERTRAM WALSH AND PETER A. LOEB
}

\author{
Communicated by R. Arens, March 25, 1966
}

1. Introduction. The axiomatic approach to potential theory instituted by Brelot [3] is well known; it abstracts in an elegant manner the properties of harmonic functions which underlie much of classical potential theory and-from a more utilitarian viewpoint-reduces the question of determining whether elliptic equations with certain classes of coefficients have a boundary-value problem theory resembling that of the Laplace equation to a few more tractable questions of an essentially local character. The purpose of this note is to announce that certain properties of the linear spaces of solutions of elliptic equations with highly differentiable coefficients (properties which bear on the construction of kernel functions) are also present in the axiomatic settings, as are certain types of behavior at ideal boundaries. We list only the main results; subsidiary results and proofs will be given elsewhere.

2. Nuclearity. Establishment of the nuclearity results does not require the full power of the Brelot axioms. Let $W$ be a locally compact Hausdorff space, and let there be given a set $\mathfrak{H C}$ of continuous realvalued functions on $W$ satisfying the following (sheaf) axiom:

I. The domains of elements of $\mathfrak{F}$ are open subsets of $W$; each $f \in \mathfrak{F C}$ is continuous on its domain; for fixed open $\Omega \subseteq W$ the set $\mathcal{K}_{\Omega}=\{f \mid f \in \mathfrak{H}$, Domain $(f)=\Omega\}$ is a real vector space, and a function $g$ with open domain $\Omega \subset W$ belongs to $\mathfrak{H}$ iff for each $x \in \Omega, \exists h \in \mathcal{F}$ and open $\omega$ with $x \in \omega \subseteq \Omega$ such that $\left.g\right|_{\omega}=\left.h\right|_{\omega}$.

Given two classes $\Re \mathcal{C}$ and $\Re$ satisfying I above, we shall say $\nVdash$ is a subclass of $\mathfrak{H}$ iff $\mathscr{K} \subseteq \mathcal{K}$; this is equivalent to saying that for any open $\Omega \subseteq W$ the vector space $\mathfrak{K}_{\Omega}$ is a subspace of $\mathfrak{K}_{\Omega}$.

For open $\Omega \subseteq W$ let $\mathfrak{H C}_{\Omega^{c}}$ denote the vector subspace of $\mathfrak{H C}_{\Omega}$ consisting of those functions which have (necessarily unique) continuous extensions to $\bar{\Omega}$. A relatively compact open subset $\Omega$ will be said to be regular (with respect to $\mathcal{F}$ ) if there exists a subset $\delta \Omega \subseteq \bar{\Omega}$ with the property that the restriction mapping $\left.f \rightarrow f\right|_{\delta \Omega}$ of $\mathcal{F C}_{\Omega}^{c}$ into $\mathfrak{C}(\delta \Omega)$ is an order-preserving isomorphism (onto); while the uniqueness of $\delta \Omega$ is not important in what follows, conditions can be given which insure

${ }_{1}^{1}$ The preparation of this paper was sponsored in part by N.S.F. Grants GP-4563 and GP-5279 respectively. 
that it is a uniquely determined subset of the Silov boundary of $\bar{\Omega}$ with respect to $\mathfrak{K}_{\Omega}^{c}$; see [1]. The classes $\mathfrak{H C}$ we shall consider satisfy local regularity and monotone-completeness conditions, summarized in the following axioms:

II. There is a base for the topology of $W$ consisting of $\mathfrak{H C}$-regular open sets.

III. For every open $\Omega \subseteq W$ and $x \in \Omega$ there exists a neighborhood $\omega$ with $x \in \omega \subset \Omega$, having the property that if $\mathscr{J}$ is a subset of $\mathfrak{F}$ and $\mathcal{J}$ is directed upward under pointwise order, then $\sup _{\mathfrak{g}} f(x)<\infty$ if and only if $\left.\left(\sup _{\mathcal{J}} f\right)\right|_{\omega} \in \mathcal{F}_{\omega} .^{2}$

Let it be agreed that any $\mathfrak{e}(X)$, where $X$ is a locally compact Hausdorff space, will be topologized by uniform convergence on compacta in $X$; then one may talk about its closed subsets. We observe that axiom II above implies that for any open $\Omega \subseteq W, \mathfrak{H}_{\Omega}$ is closed; a class $\mathfrak{H C}$ for which every $\mathfrak{H}_{\Omega}$ is closed will be called complete.

THEOREM 1. If $\Omega$ is a regular open set for a complete class Fe satisfying I and III, then for any open $\Omega_{1}$ and $\Omega_{2}$ with $\Omega_{2} \supseteq \bar{\Omega} \supseteq \Omega \supseteq \bar{\Omega}_{1}$, the restriction mapping from $\mathcal{H C}_{\Omega_{2}}$ to $\mathcal{H C}_{\Omega_{1}}$ is an integral linear transformation [5, Chapter I, pp. 126-7]; if $\Omega$ is $\sigma$-compact, then the restriction mapping $\mathfrak{H C}_{\Omega_{2}} \rightarrow \mathfrak{H C}_{\Omega}$ is integral.

Corollary (Harnack; cf. [8]): If Te satisfies I, II and III, then for any open $\Omega$ and $x_{0} \in \Omega$, the set $\left\{f \mid f \in \mathcal{F}_{\Omega}, f \geqq 0, f\left(x_{0}\right)=1\right\}$ is equicontinuous in a neighborhood $\omega$ of $x_{0}$.

In many interesting cases the following condition holds in certain open subsets $\Omega \subseteq W$ :

IV. There exists a family of regular open subsets of $\Omega$, directed upward under inclusion, whose union is $\Omega$.

TheORem 2. If He satisfies I, II and III and $\Omega$ is $\sigma$-compact, or if $\mathfrak{H C}$ is complete and satisfies I and III while $\Omega$ satisfies IV, then $\mathcal{H C}_{\Omega}$ is nuclear [5, Chapter II, p. 34].

CoRollary. Every closed subspace of such an $\mathfrak{H}_{\Omega}$ is nuclear; the dual of any metrizable closed subspace (or of any subspace if $\Omega$ is $\sigma$-compact) is nuclear.

Recall that a locally convex space is nuclear iff every continuous linear mapping from it into a Banach space can be put in the form $x \rightarrow \sum_{i=1}^{\infty} \lambda_{i}\left\langle x, x_{i}^{\prime}\right\rangle y_{i}$, where $\sum_{i=1}^{\infty}\left|\lambda_{i}\right|<\infty,\left\{x_{i}^{\prime}\right\}_{i=1}^{\infty}$ is an equicontinuous subset of the dual of the domain, and $\left\{y_{i}\right\}_{i=1}^{\infty}$ is a sequence

2 This assumption implies that $W$ is locally connected. See [2]. 
in the range with $\left\|y_{i}\right\| \rightarrow 0$; mappings of this form are called nuclear. Thus

Corollary. If $E$ is a metrizable closed subspace of $\mathfrak{H C}_{\Omega}, F$ a Banach space and $u: F \rightarrow E$ a continuous linear transformation, then there exists a sequence $\left\{\lambda_{i}\right\}_{i=1}^{\infty}$ with $\sum_{i=1}^{\infty}\left|\lambda_{i}\right|<\infty$, a sequence $\left\{h_{i}\right\}_{i=1}^{\infty} \subseteq E$ tending to zero in its topology (uniformly on compacta) and a sequence $\left\{x_{i}^{\prime}\right\}_{i=1}^{\infty} \subseteq F^{\prime}$ with $\left\|x_{i}^{\prime}\right\| \rightarrow 0$ such that for any $f \in F$

$$
u(f)=\sum_{i=1}^{\infty} \lambda_{i}\left\langle f, x_{i}^{\prime}\right\rangle h_{i} .
$$

In particular if $F=L^{1}(\mu)$ for some measure $\mu$ on a locally compact Hausdorff space $S$, then there is a sequence $\left\{g_{i}\right\}_{i=1}^{\infty} \subseteq \mathcal{L}^{\infty}(\mu)$ (functions, not classes) tending to zero uniformly on $S$ such that if

$$
G(x, s)=\sum_{i=1}^{\infty} \lambda_{i} g_{i}(s) h_{i}(x) \quad(x, s) \in \Omega \times S
$$

then $u f(x)=\int G(x, s) f(s) d \mu(s)$. Note that $G(\cdot, s) \in E$ for all $s \in S$.

Proof follows immediately from reflexivity of $E$ and nuclearity of $E_{b}^{\prime}$.

Given a harmonic class $\mathfrak{H C}$ and a complete locally convex vector space $F$, it may be desirable to define a class of $F$-valued functions which belong to $\mathfrak{H C}$ in some sense. The weakest sense in which an $F$ valued function on an open $\Omega \subseteq W$ might be said to be "Fe-harmonic" is that for each $y^{\prime} \in F^{\prime}$ the real-valued function $x \rightarrow\left\langle f(x), y^{\prime}\right\rangle$ belong to $\mathfrak{F}_{\Omega}$; on the other hand, one would want the class of $\mathfrak{F}$-harmonic $F$-valued functions to include all functions which are uniform limits on compacta of functions of the form $x \rightarrow \sum_{i=1}^{n} h_{i}(x) \cdot y_{i}$, where $h_{i} \in \mathcal{J C}_{\Omega}$ and $y_{i} \in F$. Both these senses of being $\mathfrak{H}$-harmonic coalesce for $\mathfrak{H C}$ satisfying the hypotheses of Theorem 2 above, or more generally for complete subclasses of such classes:

Proposition 1. If He satisfies I, II and III and $\Omega$ is $\sigma$-compact, or if 3e is complete and satisfies I and III while $\Omega$ satisfies IV, then for any subspace $E \subset H_{\Omega}$ and complete locally convex $F$ a function $f: \Omega \rightarrow F$ is a uniform limit on compacta in $\Omega$ of functions of the form $x \rightarrow \sum_{j=1}^{n} h_{j}(x) y_{j}$ if and only if for every $y^{\prime} \in F^{\prime}$ the function $x \rightarrow\left\langle f(x), y^{\prime}\right\rangle$ belongs to $E$.

In the axiomatic potential theory of Brelot, $W$ is taken to be noncompact, connected and locally connected; every point is assumed to have a neighborhood basis of connected open sets which satisfy II with $\delta \Omega=\partial \Omega$, the topological boundary of $\Omega$. I and III are always 
assumed; IV is implied by the requirement that there exist a positive superharmonic function on $\Omega$ (see [7]). Thus Theorem 2 holds in the Brelot situation, and the corollary of Theorem 1 becomes a recent result of the authors.

3. Ideal boundaries. Henceforth we assume the Brelot axioms (see the paragraph immediately preceding). A compactification of $W$ is a compact Hausdorff space $W^{*}$ containing $W$ as a dense open subspace; a compactification $W^{*}$ is said to be resolutive iff for every $f \in \mathcal{e}(\Delta)$, where $\Delta=W^{*} \sim W$, the lower envelope $H^{-}(f)$ of all HC-superharmonic functions $v$ with lim inf $v \geqq f$ at $\Delta$ equals the upper envelope $H_{-}(f)$ of all HC-subharmonic functions $u$ with lim sup $u \leqq f$ at $\Delta ; H(f)$ is the common value of $H_{-}(f)$ and $H^{-}(f)$ when they are equal. For resolutive compactifications, the second corollary of Theorem 2 becomes the following version of a recent result of $M$. Nakai for Riemann surfaces [9]:

Proposition 2. Let $W$ be a locally compact, noncompact, connected and locally connected Hausdorff space, countable at $\infty$, and He a harmonic class satisfying I, II and III above with $\delta=\partial$. Let $W^{*}$ be a resolutive compactification of $W$ and $\rho$ be harmonic measure on $\Delta$ with base point $x_{0}$, i.e. $\rho(f)=H(f)\left(x_{0}\right)$. Then there exists a function $G(x, s)$ $=\sum_{i=1}^{\infty} \lambda_{i} h_{i}(x) g_{i}(s)$ with $\sum_{i=1}^{\infty}\left|\lambda_{i}\right|<\infty,\left\{g_{i}\right\}_{i=1}^{\infty}$ in $\mathfrak{L}^{\infty}(\rho)$ tending to zero uniformly on $\Delta,\left\{h_{i}\right\}_{i=1}^{\infty}$ in $\mathfrak{H}_{W}$ tending to zero uniformly on compacta in $W$, such that $H(f)(x)=\int f(s) G(x, s) d \rho(s)$ for each $f \in \mathfrak{e}(\Delta)$. Moreover, there exists an increasing sequence $\left\{K_{n}\right\}_{n=1}^{\infty}$ of compact subsets of $\Delta$ for which $\bigcup_{n=1}^{\infty} K_{n}$ has $\rho$-null complement and such that $G$ is continuous on $W \times K_{n}$ for each $n$. Any two such $G$ differ at most on $W \times N$, where $N$ is $\rho$-null.

Now we suppose, in addition to the Brelot axioms, that the function 1 is 3 -superharmonic, and consider the $Q$-compactification $[4$, p. $96 \mathrm{ff}.]^{3} W^{*}$ of $W$ with $Q=B \mathcal{H}$, the (Banach) space of bounded functions in $\mathfrak{H}_{W} ; W^{*}$ is determined up to homeomorphism by the properties: $W^{*}$ is a compactification of $W$, each $h \in B \mathcal{F}$ has a unique extension in $\mathfrak{e}\left(W^{*}\right)$, and the Banach subspace of $\mathfrak{e}\left(W^{*}\right)$ consisting of those extensions separates points of $\Delta=W^{*} \sim W$. One may now show that the cone of positive elements of BFC has a Silov boundary $\kappa \subseteq W^{*}$, that $\kappa \subseteq \Delta$, and that $\left.B \mathcal{H C}\right|_{\kappa}=\mathfrak{C}(\kappa)$. Using a generalization of a barrier argument developed in [6, pp. 44-45] we show that the

3 [4] treats $Q$-compactifications only for Riemann surfaces; however, it is easy to verify that their construction and characterization make use only of the local compactness of the surface. 
points of $\kappa$ are regular in the sense that for each point $s \in \kappa$ and every bounded function $f$ on $\Delta$

$$
\liminf _{\Delta} f(s) \leqq \liminf _{W} H_{-}(f)(s) \leqq \limsup _{W} B^{-}(f)(s) \leqq \lim _{\Delta} \sup f(s)
$$

(where the subscripts denote the sets through which $s$ is approached, and the point $s$ itself is not excluded). It can now be shown that $W^{*}$ is resolutive, and further

THEOREM 3. Let $\rho$ be harmonic measure on $\Delta$ with base point $x_{0} \in W$. There is an unique function $G \in \mathfrak{C}\left(W \times W^{*}\right)$ such that $\left.G\right|_{W \times W}$ is $\mathfrak{H}$ harmonic in each variable separately and $H(f)=\int f(s) G(\cdot, s) d \rho(s)$ for any $f \in \mathfrak{C}(\Delta)$.

REMARK. H. S. Bear has kindly informed one of the authors that he and A. M. Gleason have obtained results on the existence of kernel functions and measures in a setting somewhat different from ours. An abstract of their work is to be found in [A].

\section{REFERENCES}

1. H. Bauer, Šilovscher Rand und Dirichletsches Problem, Ann. Inst. Fourier (Grenoble) 11 (1961), 89-136.

2. N. Boboc, C. Constantinescue and A. Cornea, Axiomatic theory of harmonic functions. Non-negative superharmonic functions. Ann. Inst. Fourier (Grenoble) (1) 15 (1965), 283-312.

3. M. Brelot, Lectures on potential theory, Tata Institute of Fundamental Research, Bombay, 1960.

4. C. Constantinescu and A. Cornea, Ideale Ränder Riemannscher Flächen, Ergebnisse der Mathematik, neue Folge, Bd. 32, 1963.

5. A. Grothendieck, Produits tensoriels topologiques et espaces nucleaires, Mem. Amer. Math. Soc. No. 16 (1955), 191+140 pp.

6. P. A. Loeb, An axiomatic treatment of pairs of elliptic differential equations, Doctoral Dissertation, Stanford Univ., 1963.

7. - - An axiomatic treatment of pairs of elliptic differential equations, Ann. Inst. Fourier (Grenoble) (to appear).

8. P. A. Loeb and B. Walsh, The equivalence of Harnack's principle and Harnack's inequality in the axiomatic system of Brelot, Ann. Inst. Fourier (Grenoble) 15 (1965), $597-600$.

9. M. Nakai, Radon-Nikodym densities between harmonic measures on the ideal boundary of an open Riemann surface, Nagoya Math. J. 27 (1965), 71-76.

A. H. S. Bear and A. M. Gleason, An integral formula for abstract harmonic or parabolic functions, Abstract 633-1, Notices Amer. Math. Soc. 13 (1966), 348.

University of California, Los Angeles 